\title{
Monopole field textures in interacting spin systems
}

\author{
Andreas Eriksson and Erik Sjöqvist (1)* \\ Department of Physics and Astronomy, Uppsala University, Box 516, Se-751 20 Uppsala, Sweden
}

(Received 13 March 2020; accepted 6 May 2020; published 29 May 2020)

\begin{abstract}
Magnetic monopoles can appear as emergent structures in a wide range of physical settings, ranging from spin ice to Weyl points in semimetals. Here, a distribution of synthetic (Berry) monopoles in parameter space of a slowly changing external magnetic field is demonstrated in a system of interacting spin- $\frac{1}{2}$ particles with broken spherical symmetry. These monopoles can be found at points where the external field is nonzero. The spin-spin interaction provides a mechanism for splitting the synthetic local magnetic charges until their magnitude reaches the smallest allowed value $\frac{1}{2}$. For certain states, a nonzero net charge can be created in an arbitrarily large finite region of parameter space. The monopole field textures contain nonmonopolar contributions in the presence of spin-spin interaction.
\end{abstract}

DOI: 10.1103/PhysRevA.101.050101

While magnetic monopoles seem up to this date mysteriously absent as fundamental entities in nature, they may occur as emergent structures in various physical systems. Indeed, real-space realizations of such emergent monopoles have been demonstrated in spin ice [1] and Bose-Einstein condensates [2], but also in reciprocal space of crystalline systems, e.g., in the context of anomalous Hall effect [3], as well as in the form of Weyl points in semimetals [4] and photonic crystals [5].

More generally, magnetic monopoles are ubiquitous in parameter spaces of adiabatic systems, as demonstrated by Berry [6]. Perhaps most well known is the canonical example of a single spin in a slowly changing external magnetic field, as described by the Zeeman interaction. Due to the spherical symmetry of this system, the monopole is forced to the origin of parameter space, where the external magnetic field vanishes. The corresponding magnetic charge is essentially the quantum number along the quantization axis of the instantaneous spin eigenstate. Such synthetic magnetic monopoles in spinlike systems have been studied experimentally in a wide range of settings [7-12].

Here, we examine magnetic monopoles in spin systems with broken spherical symmetry. Specifically, we provide a proof-of-concept demonstration of monopole field textures in the simplest nontrivial case consisting of a pair of interacting spins. Our purpose is to demonstrate a mechanism for how tunable magnetic monopole structures can be created in spin composites exposed to slowly varying external magnetic fields.

\footnotetext{
*erik.sjoqvist@physics.uu.se
}

Published by the American Physical Society under the terms of the Creative Commons Attribution 4.0 International license. Further distribution of this work must maintain attribution to the author(s) and the published article's title, journal citation, and DOI. Funded by Bibsam.
Our system consists of two identical spin- $\frac{1}{2}$ particles in a slowly changing external magnetic field $\mathbf{b}$. The two spins $\mathbf{s}_{1}$ and $\mathbf{s}_{2}$ are coupled by a nonzero uniaxial exchange (Ising) interaction in the $z$ direction, combined with a DzyaloshinskiiMoriya interaction (DMI) term. The Hamiltonian reads

$$
H(\mathbf{b} ; J, \mathbf{D})=\mathbf{b} \cdot \mathbf{S}+4 J s_{1}^{z} s_{2}^{z}+\mathbf{D} \cdot\left(\mathbf{s}_{1} \times \mathbf{s}_{2}\right)
$$

with $\mathbf{S}=\mathbf{s}_{1}+\mathbf{s}_{1}$ the total spin and $J$ the Ising coupling strength. The DMI vector $\mathbf{D}$ is confined to the $b_{x} b_{z}$ plane, i.e., $\mathbf{D}=D(\sin \vartheta, 0, \cos \vartheta)$ with $\vartheta$ the angle between the DMI and Ising axes [13]. For notational convenience, we represent the spin-spin coupling by the vector $\vec{g}=(J, \mathbf{D})$. The system possesses cylindrical symmetry in the special case where $\vec{g}=$ $(J, 0,0, D)$; full rotation symmetry is restored when $\vec{g}=\overrightarrow{0}$. In the general case with $J, D, \sin \vartheta \neq 0$, both these symmetries are broken.

In parameter space defined by the external field $\mathbf{b}$, there is a synthetic magnetic field [6]

$$
\boldsymbol{B}^{(k)}(\mathbf{b} ; \vec{g})=i \sum_{l \neq k} \frac{\mathbf{S}_{k l}(\mathbf{b} ; \vec{g}) \times \mathbf{S}_{l k}(\mathbf{b} ; \vec{g})}{\left[E_{l}(\mathbf{b} ; \vec{g})-E_{k}(\mathbf{b} ; \vec{g})\right]^{2}}
$$

associated with each energy eigenstate $\left|\psi_{k}(\mathbf{b} ; \vec{g})\right\rangle, \quad k=$ $1, \ldots, 4$, where $\quad \mathbf{S}_{k l}(\mathbf{b} ; \vec{g})=\left\langle\psi_{k}(\mathbf{b} ; \vec{g})|\mathbf{S}| \psi_{l}(\mathbf{b} ; \vec{g})\right\rangle$. These fields define monopole charges $q_{\mu}^{(k)}$ that are located at energy crossing points $\mathbf{b}_{\mu}^{(k)}$. The charges are computed by means of Gauss' law

$$
\frac{1}{4 \pi} \iint_{\partial V} \boldsymbol{B}^{(k)}(\mathbf{b} ; \vec{g}) \cdot d \mathbf{S}=\sum_{\mu \in V} q_{\mu}^{(k)},
$$

where $V$ is a finite volume enclosed by a smooth orientable surface $\partial V$ in parameter space.

The following sum rules are useful for analyzing the monopole distribution of the system. First,

$$
\sum_{k} \sum_{\mu \in V} q_{\mu}^{(k)}=0
$$



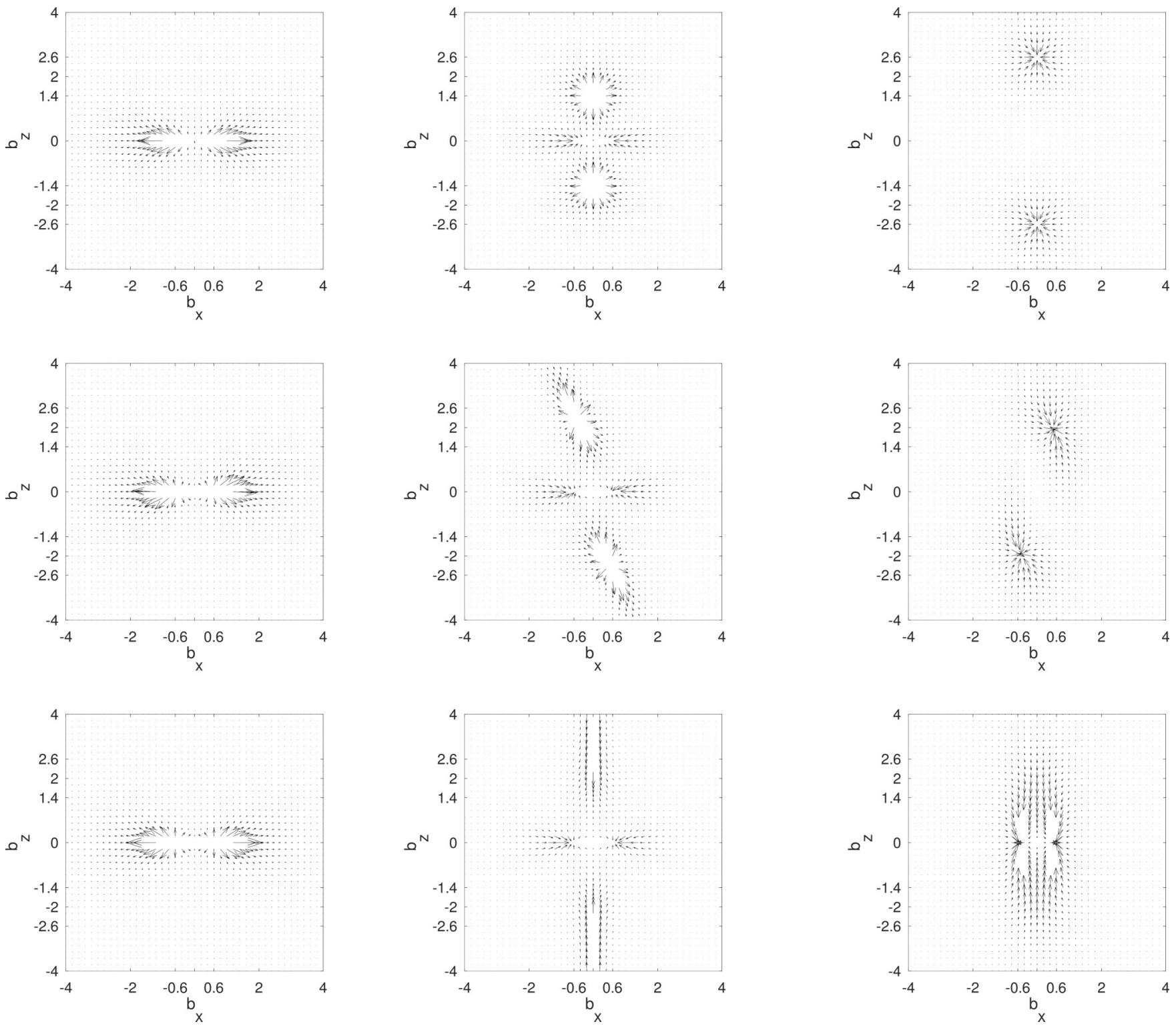

FIG. 1. Two-dimensional cuts of the synthetic magnetic fields for $J=1$ and $D=0.3$ with $b_{x}$ and $b_{z}$ shown in units of $J$. From left to right in each row are shown $\mathbf{B}^{(1)}, \mathbf{B}^{(2)}$, and $\mathbf{B}^{(3)}$ with total magnetic charges $Q^{(1)}=+1, Q^{(2)}=0$, and $Q^{(3)}=-1$, respectively. Upper row shows $\vartheta=0^{\circ}$, where monopoles are expected at the energy crossing points that are found at $(0,0,0),[0,0, \pm(2 J+2 D)]=(0,0, \pm 2.6)$, and $[0,0, \pm(2 J-2 D)]=(0,0, \pm 1.4)$. Middle row shows $\vartheta=60^{\circ}$. Lower row shows $\vartheta=90^{\circ}$ with all monopoles at $( \pm 2 D, 0,0)=( \pm 0.6,0,0)$ Note that there is a nonzero magnetic charge $q_{+}^{(2)}+q_{-}^{(2)}=-1$ associated with $\mathbf{B}^{(2)}\left(\vartheta=90^{\circ}\right)$ as two monopoles, each with charge $+\frac{1}{2}$, have been moved to infinity for this angle. Note that large arrows in the plot of $\mathbf{B}^{(2)}\left(\vartheta=90^{\circ}\right)$ have been omitted along the $b_{z}$ axis for magnetic field strengths $\left|b_{z}\right|>2$, in order to avoid obscuring the neighboring arrows.

for any $V$, which can be seen by combining the identity [14]

$$
\sum_{k} \boldsymbol{B}^{(k)}(\mathbf{b} ; \vec{g})=0
$$

with Eq. (3). Secondly, the total magnetic charge

$$
Q^{(k)}=\sum_{\mu} q_{\mu}^{(k)}
$$

for a given state $\psi_{k}$ is preserved under changes of $\vec{g}$. This follows from the observation that pairwise crossings of states occur at hypersurfaces of codimension 3 in the extended parameter space $(\mathbf{b}, J, \mathbf{D})$. According to the von NeumannWigner theorem [15], this implies that independent variation of the three components of $\mathbf{b}$ is sufficient to induce pointlike energy level crossings in the parameter space of the slowly changing magnetic field, no matter the form of spin-spin interaction. The total charge $Q^{(k)}$ is thus a topological invariant of the state $\psi_{k}$.

We are now prepared to examine the synthetic magnetic fields in the spin-pair system. To determine the total magnetic charges, we first consider the pure Zeeman case $(J=D=0)$. Here, the energy eigenstates coincide with the singlet-triplet states $|S, M ; \mathbf{n} \cdot \mathbf{S}\rangle, S=0,1, M=-S, \ldots, S$, along the direction $\mathbf{n}=\mathbf{b} /|\mathbf{b}|$ of the instantaneous external magnetic field. As shown by Berry [6], one obtains $\mathbf{B}^{(k)}(\mathbf{b} ; \overrightarrow{0}) \equiv \mathbf{B}^{(M)}(\mathbf{b} ; \overrightarrow{0})=$ $-M \mathbf{b} /|\mathbf{b}|^{3}$, which is a purely monopolar field. Thus, each 

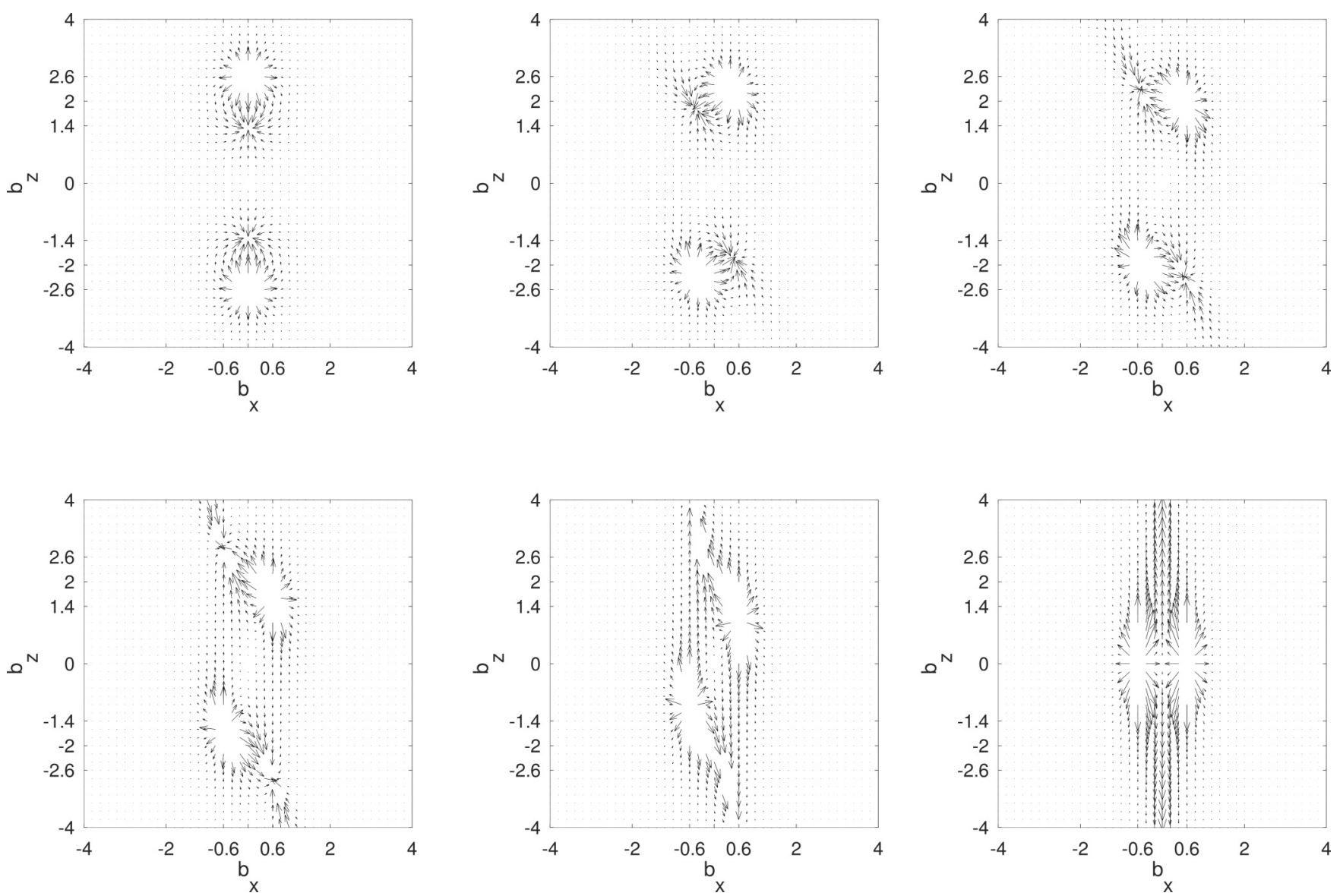

FIG. 2. Two-dimensional cuts of $\mathbf{B}^{(4)}(\mathbf{b} ; J=1, D=0.3, \vartheta)$ for rotated DMI with $b_{x}$ and $b_{z}$ shown in units of $J$. From upper left to lower right: $\vartheta=0^{\circ}, 45^{\circ}, 60^{\circ}, 70^{\circ}, 80^{\circ}, 90^{\circ}$. The net magnetic charge vanishes for all $\vartheta \neq 90^{\circ}$. The progression to the net charge $q_{+}^{(4)}+q_{-}^{(4)}=+1$ by moving two monopoles, each with charge $-\frac{1}{2}$, to infinity, is visible.

synthetic magnetic field $\mathbf{B}^{(M)}$ correspond to a total magnetic monopole charge $Q^{(k)} \equiv Q^{(M)}=-M$ located at the origin of parameter space.

Next, we add a nonzero Ising term, while keeping a vanishing DMI $(J \neq 0, D=0)$. The spin singlet remains decoupled from the triplet states, and its corresponding synthetic magnetic field therefore vanishes. Due to the cylindrical symmetry of the system, the energies are independent of the azimuthal spherical angle, which implies that the monopoles must be located on the $b_{z}$ axis [16]. Indeed, by diagonalizing the Hamiltonian in the triplet subspace, one finds intersection points only at $\mathbf{b}_{0}^{(k)}=(0,0,0)$ and $\mathbf{b}_{ \pm}^{(k)}=(0,0, \pm 2 J)$ in parameter space. The corresponding magnetic charges $q_{0}^{(k)}$ and $q_{ \pm}^{(k)}$ can be found by numerically performing the integration in Eq. (3) around each of these intersection points. One finds

$$
\begin{aligned}
& q_{0}^{(1)}=+1 ; \quad q_{0}^{(2)}=-1, \\
& q_{+}^{(2)}=q_{-}^{(2)}=+\frac{1}{2} ; \quad q_{+}^{(3)}=q_{-}^{(3)}=-\frac{1}{2},
\end{aligned}
$$

which confirm the sum rules in Eqs. (4) and (6). Note the appearance of half-integer magnetic charges for $\psi_{2}$ and $\psi_{3}$.

We now turn to the general case where both $J$ and $D$ are nonvanishing. The broken symmetry caused by rotating D creates a nontrivial pattern of intersections of all four states, which in turn shows up as a nontrivial distribution of magnetic charges. Figure 1 shows the synthetic fields $\mathbf{B}^{(k)}$, $k=1,2,3$, each for $\vartheta=0^{\circ}, 60^{\circ}, 90^{\circ}$. For clarity, we show two-dimensional cuts of the field textures that contain the monopole charges. The remaining integer valued charges of $\psi_{1}$ and $\psi_{2}$ at the origin are now split into pairs of half-integer charges for $\vartheta \neq 0^{\circ}$. We note that no further splitting can take place by introducing other types of spin-spin interaction terms in the Hamiltonian, since the magnitude of each charge is $\frac{1}{2}$, which is the smallest allowed value [6]. The cylindrical symmetry still holds for $\vartheta=0^{\circ}$, which forces the monopoles to remain on the $b_{z}$ axis in this case. For $\vartheta \neq 0^{\circ}$, the symmetry is lowered and the magnetic charges move into the $b_{x} b_{z}$ plane, but differently for $\psi_{2}$ and $\psi_{3}$, thereby creating a nonzero local total magnetic charge in the $\left\{\psi_{1}, \psi_{2}, \psi_{3}\right\}$ manifold. These nonzero local net charges are exactly canceled by the local magnetic charges of $\psi_{4}$, as shown in Fig. 2, which confirms the sum rule in Eq. (4).

By numerically computing the flux of $\mathbf{B}^{(4)}$ through a surface $\partial V$ that encloses the two magnetic charges at $\mathbf{b}_{ \pm}^{(4)}=$ $( \pm 2 D, 0,0)$ for $\vartheta=90^{\circ}$, one finds the net magnetic charge $q_{+}^{(4)}+q_{-}^{(4)}=+1$, no matter the size of the region $V$ bounded by $\partial V$. Thus, a nonzero magnetic charge has been created. This is still consistent with the sum rule in Eq. (6), as two monopoles, each with charge $-\frac{1}{2}$, have been moved to infinity when the DMI vector is rotated towards $\vartheta=90^{\circ}$. To clearly show this progression, we have included the intermediate 
cases $\vartheta=45^{\circ}, 70^{\circ}, 80^{\circ}$ in Fig. 2. As can be seen in Fig. 1, there is a nonzero net magnetic charge $q_{+}^{(2)}+q_{-}^{(2)}=-1$ for $\psi_{2}$ at $\vartheta=90^{\circ}$. Just as for $\psi_{4}$, these charges are located at $( \pm 2 D, 0,0)$, which again confirms Eq. (4).

Contrary to the Zeeman case, the synthetic magnetic field textures shown in Figs. 1 and 2 are not purely monopolar in the presence of spin-spin interaction, i.e., it can be verified that

$$
\mathbf{B}^{(k)}(\mathbf{b} ; \vec{g} \neq \overrightarrow{0}) \neq \sum_{\mu} q_{\mu}^{(k)} \frac{\mathbf{b}-\mathbf{b}_{\mu}^{(k)}}{\left|\mathbf{b}-\mathbf{b}_{\mu}^{(k)}\right|^{3}}
$$

Stated differently, the synthetic "electrical current density" defined via the Ampere-Maxwell-type equation $\mathbf{j}^{(k)}(\mathbf{b} ; \vec{g})=$ $\nabla \times \mathbf{B}^{(k)}(\mathbf{b} ; \vec{g})$ is nonvanishing for $\vec{g} \neq \overrightarrow{0}$.

By extending the system to more than two interacting spins, the total charges $Q^{(k)}$ become integer or half-odd integer valued depending on whether the system contains an even or odd number of spins, respectively. For such multispin systems, more complex pointlike structures of an increasing number of monopoles are expected, as the total charges $Q^{(k)}$ can take increasingly larger values with the number of involved spins.
In conclusion, we have provided a proof-of-concept demonstration of nontrivial magnetic monopole structures in a system of two interacting spin- $\frac{1}{2}$ particles. These monopoles can appear at points in parameter space where the external magnetic field is nonzero. This is in sharp contrast to the pure Zeeman case where monopoles only can appear at vanishing external magnetic field. We have shown that by increasing the complexity of the spin-spin interaction, the magnetic charges can be split into smaller entities. Furthermore, a nonzero magnetic charge can be created by tuning the spinspin interaction for certain states. The synthetic magnetic field textures are nonmonopolar, which correspond to a nonvanishing synthetic electrical current density in parameter space. Our findings show that systems of interacting spins can give rise to highly nontrivial magnetic monopole structures. Trajectories of particles composed of interacting spins and moving in spatially inhomogeneous external magnetic fields are sensitive to the Lorentz-type forces induced by the monopoles and synthetic electric currents. This feature provides a tool for studying the synthetic magnetic field structure experimentally.

E.S. acknowledges support from the Swedish Research Council (VR) under Grant No. 2017-03832.
[1] C. Castelnovo, R. Moessner, and S. L. Sondhi, Magnetic monopoles in spin ice, Nature (London) 451, 42 (2008).

[2] M. W. Ray, E. Ruokokoski, K. Tiurev, M. Möttönen, and D. S. Hall, Observation of isolated monopoles in a quantum field, Science 348, 544 (2015).

[3] Z. Fang, N. Nagaosa, K. S. Takahashi, A. Asamitsu, R. Mathieu, T. Ogasawara, H. Yamada, M. Kawasaki, Y. Tokura, and K. Terakura, The anomalous Hall effect and magnetic monopoles in momentum space, Science 302, 92 (2003).

[4] B. Q. Lv, H. M. Weng, B. B. Fu, X. P. Wang, H. Miao, J. Ma, P. Richard, X. C. Huang, L. X. Zhao, G. F. Chen, Z. Fang, X. Dai, T. Qian, and H. Ding, Experimental Discovery of Weyl Semimetal TaAs, Phys. Rev. X 5, 031013 (2015).

[5] L. Lu, Z. Wang, D. Ye, L. Ran, L. Fu, J. D. Joannopoulos, and M. Soljacić, Experimental observation of Weyl points, Science 349, 622 (2015).

[6] M. V. Berry, Quantal phase factors accompanying adiabatic changes, Proc. R. Soc. London, Ser. A 392, 45 (1984).

[7] A. Tomita and R. Y. Chiao, Observation of Berry's Topological Phase by Use of an Optical Fiber, Phys. Rev. Lett. 57, 937 (1986).

[8] T. Bitter and D. Dubbers, Manifestation of Berry's Topological Phase in Neutron Spin Rotation, Phys. Rev. Lett. 59, 251 (1987).
[9] D. Suter, G. C. Chingas, R. A. Harris, and A. Pines, Berry's phase in magnetic resonance, Mol. Phys. 61, 1327 (1987).

[10] Ch. Miniatura, J. Robert, O. Gorceix, V. Lorent, S. Le Boiteux, J. Reinhardt, and J. Baudon, Atomic Interferences and the Topological Phase, Phys. Rev. Lett. 69, 261 (1992).

[11] P. J. Leek, J. M. Fink, A. Blais, R. Bianchetti, M. Göppl, J. M. Gambetta, D. I. Schuster, L. Frunzio, R. J. Schoelkopf, and A. Wallraff, Observation of Berry's Phase in a Solid-State Qubit, Science 318, 1889 (2007).

[12] K. Arai, J. Lee, C. Belthangady, D. R. Glenn, H. Zhang, and R. L. Walsworth, Geometric phase magnetometry using a solidstate spin, Nat. Commun. 9, 4996 (2018).

[13] The combination of Ising and DMI terms is chosen so as to give rise to a nontrivial but simply analyzable pattern of magnetic monopoles. One may envisage other combinations of coupling terms that can achieve different forms of monopole structures.

[14] D. Chruscinski and A. Jamiolkowski, Geometric Phases in Classical and Quantum Mechanics (Birkhäuser, Basel, 2004).

[15] J. von Neumann and E. P. Wigner, Über das Verhalten von Eigenwerten bei adiabatischen Prozessen, Phys. Z. 30, 467 (1929).

[16] Otherwise they would, as a consequence of the cylindrical symmetry, form ringlike structures around the $b_{z}$ axis, which is not allowed by the von Neumann-Wigner theorem. 